\title{
Pharmacoresistant Atypical Benign Partial Epilepsy of Childhood: Early Introduction of Specific Therapies to Prevent Cognitive Decline
}

\author{
Aiko Nishikawa ${ }^{1}$ Yui Otani ${ }^{1}$ Susumu Ito ${ }^{1}$ Satoru Nagata ${ }^{1}$ Hirokazu Oguni ${ }^{10}$ \\ ${ }^{1}$ Department of Pediatrics, Tokyo Women's Medical University, \\ Shinjuku-ku, Tokyo, Japan \\ Address for correspondence Hirokazu Oguni, MD, Department of \\ Pediatrics, Tokyo Women's Medical University, 8-1 Kawada-Cho, \\ J Pediatr Epilepsy 2020;9:7-12. \\ Shinjuku-ku, Tokyo 162, Japan (e-mail: oguni.hirokazu@twmu.ac.jp).
}

\begin{abstract}
Atypical benign partial epilepsy (ABPE) of childhood is a special type of epileptic syndrome characterized by the combination of epileptic negative myoclonus, atypical absence seizures, focal motor seizures, and a continuous spike-wave during slow sleep (CSWS). Although the seizures are resistant to antiseizure drugs (ASDs) effective for focal epilepsy, they are markedly responsive to ethosuximide (ESM). An incorrect ASD choice may aggravate the seizures and CSWS, resulting in a pseudo-catastrophic state or nonconvulsive status epilepticus. In this study, we retrospectively investigated the effectiveness of steroid and ketogenic diet (KD) therapies for children with ABPE resistant to ASDs, including ESM. Adrenocorticotropic hormone (ACTH) therapy $(n=4)$, KD therapy $(n=4)$, and an oral steroid $(n=2)$ were tried for eight patients (two patients tried two therapies). An excellent response (seizure-free period $>1$ year)

\section{Keywords}

- atypical benign partial epilepsy

- CSWS

- ACTH therapy

- ketogenic diet

- ethosuximide was achieved by four out of four patients undergoing ACTH therapy, two out of four undergoing KD therapy, and one out of two receiving oral steroid therapy. In conclusion, steroid and KD therapies are reasonable treatment options early in the course of treatment of children with pharmacoresistant ABPE to prevent a decline in cognitive function due to the persistence of epileptic encephalopathy. The majority of patients in our case series had an excellent response, though further studies are warranted to confirm their efficacy and safety.
\end{abstract}

\section{Introduction}

Atypical benign partial epilepsy (ABPE) of childhood is a special type of epileptic syndrome first described by Aicardi and Chevrie in $1982 .^{1}$ Since then, there have been many studies characterizing the syndrome by the presence of epileptic negative myoclonus (ENM), atypical absence seizures, and centrotemporal focal epileptic electroencephalographic (EEG) abnormality progressing to a continuous spike-wave during slow sleep (CSWS). ${ }^{2-5}$ Although the seizures eventually go into remission with increasing age, they are often resistant to antiseizure drugs (ASDs) effective for focal epilepsy. In our clinical practice, we recommended avoiding carbamazepine (CBZ), and trying ethosuximide

received

July 20, 2019

accepted after revision

September 27, 2019

published online

November 7, 2019
(ESM), which can promptly control ENM as well as atypical absence seizures in most patients ( $100 \%)$ with ABPE despite its indication for focal epilepsy. ${ }^{3,5,6}$ We previously reported that children with ABPE exhibit a self-limiting clinical course with episodic aggravation and eventually enter remission in adolescence, although those refractory to standard treatment and with prolonged seizures were found to have an overall decline in cognitive function. ${ }^{5}$ Thus, early recognition and early control of the seizures using an appropriate treatment strategy are important.

However, some children with ABPE already receiving multiple ASD medications, including ESM, present with numerous ENM and atypical absence seizures as well as focal
Copyright @ 2020 by Georg Thieme Verlag KG, Stuttgart . New York
DOI https://doi.org/ 10.1055/s-0039-3399512. ISSN 2146-457X. 
motor seizures. For such conditions, we have tried steroid therapy or ketogenic diet (KD) therapy to control both the epileptic seizures and CSWS while simultaneously adjusting ASDs. ${ }^{5}$ We report eight patients with pharmacoresistant ABPE successfully undergoing these specific therapies.

\section{Patients}

We retrospectively reviewed patients with ABPE and identified eight whose seizures were resistant to ASDs and who started steroid or KD therapy. They fulfilled the clinical and EEG criteria of ABPE. Two patients received both therapies because of an unsatisfactory response to either trial. We reviewed the medical records, neuroimaging data, EEGs and other laboratory data, and their long-term clinical course. Regarding adrenocorticotropic hormone (ACTH) therapy, synthetic ACTH-Z (Cortrosyn-ZR, zinc hydroxide suspension of tetracosactrin, $1 \mathrm{mg}$ equivalent to $40 \mathrm{IU}$ ) at a dose of $0.025 \mathrm{mg} / \mathrm{kg}$ (maximum of up to $0.5 \mathrm{mg} / \mathrm{d}$ ) was injected once every morning for 2 weeks, followed by tapering over the subsequent 2 weeks. For oral steroid therapy, $2 \mathrm{mg} / \mathrm{kg}$ prednisolone or $10 \mathrm{mg} / \mathrm{kg}$ hydrocortisone was maintained for 2 weeks and tapered gradually over 1 to 2 months. KD therapy was initiated with up to 48 hours of fasting, followed either by classical KD (3:1) or medium chain triglyceride (MCT)-KD (2:1).

Regarding treatment response, excellent and good responses were defined as cessation of the seizures and resolution of CSWS, and a more than $80 \%$ reduction in the seizures and resolution of CSWS, respectively. Other responses were considered unsatisfactory. The effectiveness was assessed at 3 months (short-term response) and 1 year (long-term response) after the therapy.

\section{Representative Case Report}

\section{Case 4}

The patient was a girl aged 10 years and 6 months. She was born uneventfully at 39 weeks of gestation weighing $2,804 \mathrm{~g}$.
Her developmental milestones were normal before the onset of epilepsy. Her family history was negative for seizure disorders. At 4 years and 4 months of age, she developed febrile and afebrile focal motor seizures involving either the left or right side of the body once a month, which lasted up to 30 minutes (-Fig. 1). At a local hospital, she was administered valproic acid (VPA), CBZ, and clobazam without effect. She was referred to our hospital at 5 years and 5 months of age. Long-term video-EEG monitoring over 24 hours demonstrated frequent intermittent sharp-slow discharges predominantly from the left centroparietal regions during wakefulness, which appeared almost continuously during sleep (spike-wave index $=60 \%$ ) ( -Fig. 2A). She was unable to write or grasp objects because of paresis of the right hand due to frequent ENM manifesting with dropping of either the right or both hands. Magnetic resonance imaging of the brain was normal. As she had pharmacoresistant ABPE resulting in severe epileptic encephalopathy, we decided to introduce ACTH therapy, which immediately controlled the seizures and CSWS (-Fig. 2B, C). Since then, she had focal to bilateral tonic-clinic seizures (F-BTCS) only once a year, and was cognitively alert and able to attend regular elementary school. At 9 years and 9 months of age, she developed monthly F-BTCS, daily atypical absence seizures, and CSWS (spike-wave index $=60 \%$ ), and became mute and inattentive. At this time, we successfully introduced classical KD (3:1). Although F-BTCS transiently recurred 3 months later when ketosis was reduced, she has since been seizure free.

\section{Summary of Eight Patients}

The eight patients (10 trials) are summarized in - Table 1. The ages at epilepsy onset ranged from 24 to 76 months (median: 44 months). The ENM, atypical absence seizures, and CSWS started between 24 and 84 months of age, with a median of 60 months. The time between the onset of epilepsy and that of ABPE ranged from 0 and 42 months, with a median of 9 months. No ASDs induced the evolution from rolandic epilepsy (RE) to

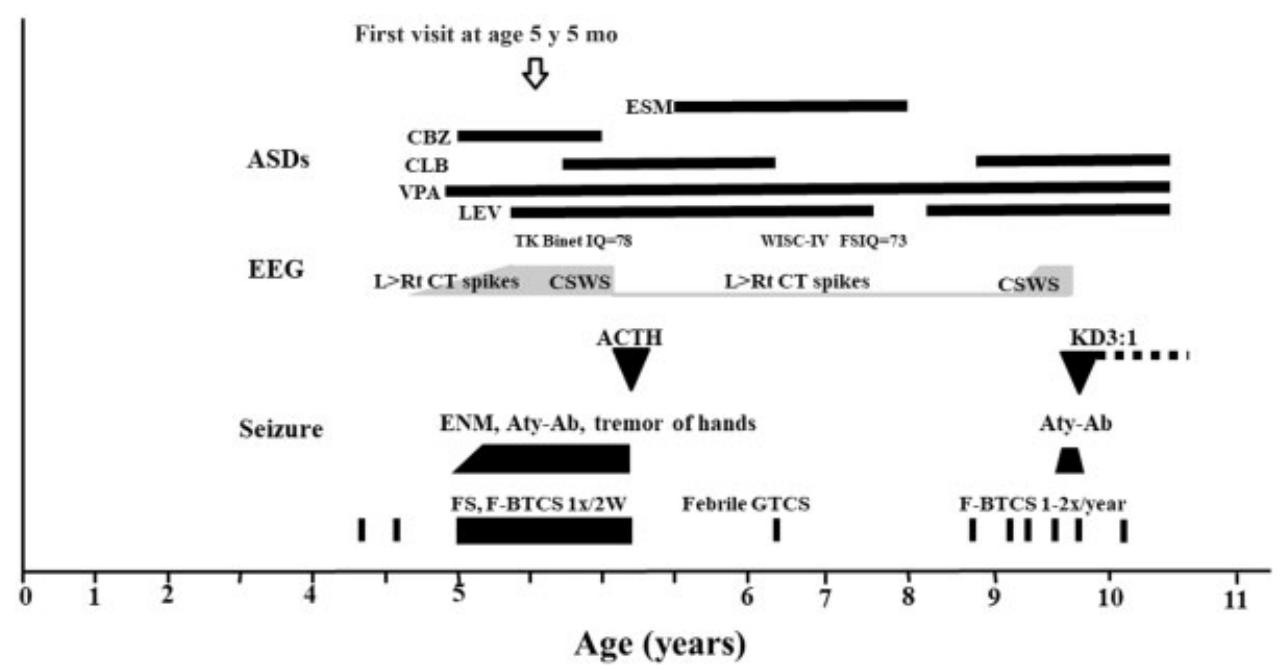

Fig. 1 Clinical course of case 4. ACTH, adrenocorticotropin; ASDs, antiseizure drugs; Atyp-Ab, atypical absence seizures; CSWS, continuous spike-waves during slow sleep; ENM, epileptic negative myoclonus; F-BTCS, focal to bilateral tonic-clonic seizures; FS, focal (motor) seizures; KD, ketogenic diet. 

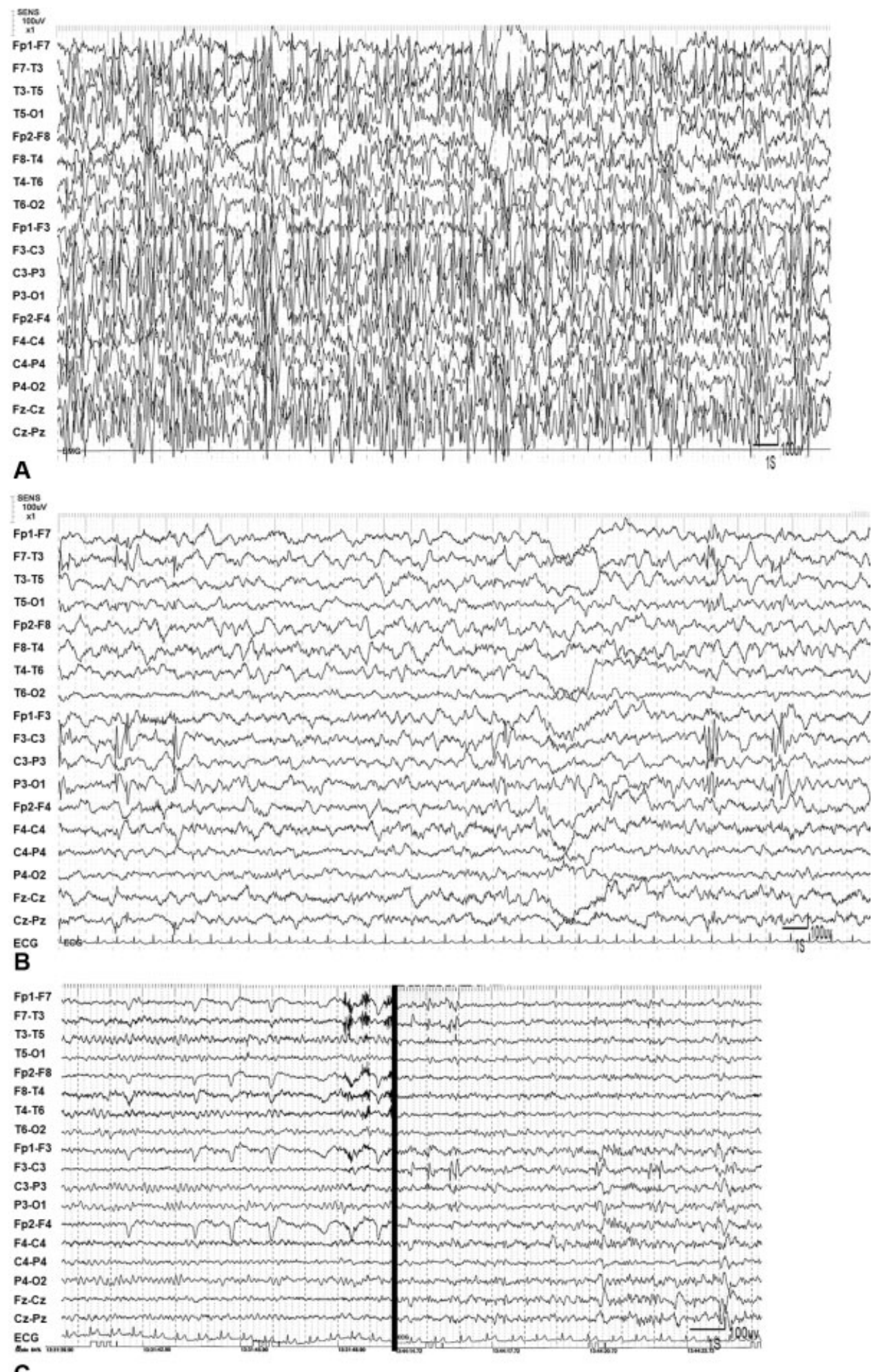

C

Fig. 2 Interictal sleep EEG before and after the introduction of ACTH therapy in case 4. (A) At 2 weeks before ACTH therapy, continuous diffuse spike-waves with left hemispheric predominance were recorded during non-REM sleep confirmed by 24-hour video-EEG monitoring (spike-wave index $=60 \%$ ). (B) Two days after the cessation of ACTH therapy, the interictal EEG markedly improved, showing infrequent low-amplitude centrotemporal sharp-and-slow wave complexes in the left hemisphere. (C) Three months after the cessation of ACTH therapy, EEG findings during wakefulness and sleep were normal except for rare left centrotemporal spike-and-wave complexes. ACTH, adrenocorticotropin; EEG, electroencephalography; REM, rapid eye movement.

ABPE. The spike-wave index of CSWS ranged from 60 to $100 \%$ in six patients undergoing overnight EEG. At the peak of epileptic encephalopathy, they frequently exhibited paresis of the hands or legs, dysarthria, ataxia, and slow and apathetic behavior. The follow-up period was between 40 and 243 months, with a median of 85 months. Decline in cognitive 


\begin{tabular}{|c|c|c|c|c|c|c|c|c|c|c|c|}
\hline 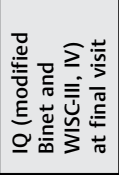 & 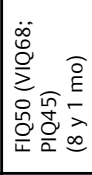 & 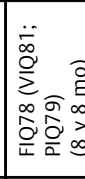 & 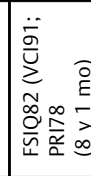 & 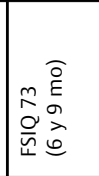 & & 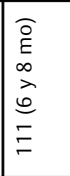 & 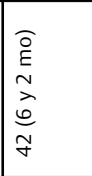 & & 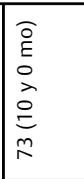 & 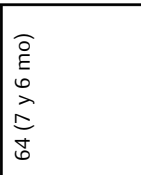 & 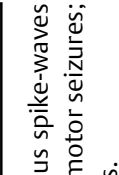 \\
\hline 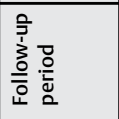 & 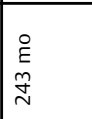 & 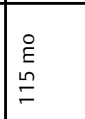 & $\mid \begin{array}{c}\stackrel{\circ}{E} \\
\underset{\infty}{E}\end{array}$ & & 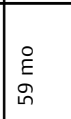 & $\begin{array}{l}\stackrel{O}{E} \\
\mathcal{F}\end{array}$ & \begin{tabular}{|l}
$O$ \\
$\stackrel{E}{\sigma}$ \\
$\sigma$
\end{tabular} & & $\mid \begin{array}{l}\stackrel{0}{E} \\
\underset{N}{N}\end{array}$ & \begin{tabular}{|l}
$\stackrel{\circ}{E}$ \\
E् \\
$\infty$
\end{tabular} & 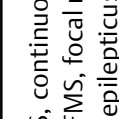 \\
\hline 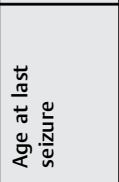 & $\begin{array}{l}\stackrel{0}{E} \\
\frac{1}{\lambda} \\
\end{array}$ & $\begin{array}{l}0 \\
E \\
\infty \\
0 \\
0\end{array}$ & 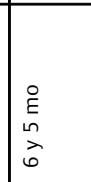 & 1 & 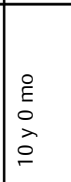 & $\begin{array}{l}0 \\
E \\
0 \\
\\
0 \\
0\end{array}$ & 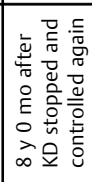 & 1 & 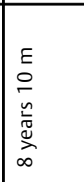 & 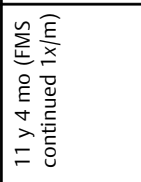 & 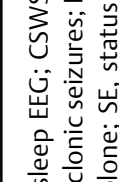 \\
\hline 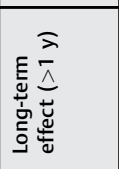 & 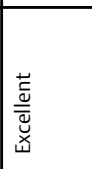 & 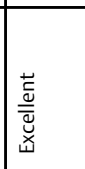 & 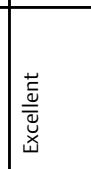 & 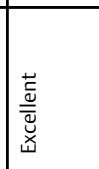 & 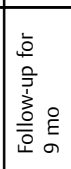 & \begin{tabular}{|l}
$\overrightarrow{\vec{v}}$ \\
$\frac{\vec{v}}{\overline{\tilde{U}}}$ \\
$\breve{v}$
\end{tabular} & 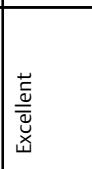 & 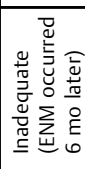 & 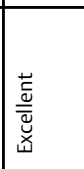 & 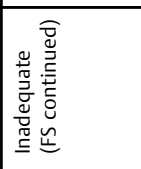 & 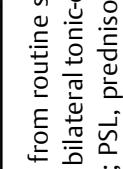 \\
\hline 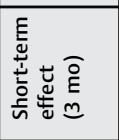 & 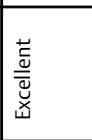 & 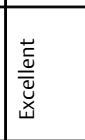 & 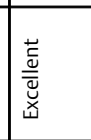 & 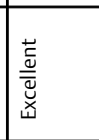 & ס & 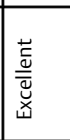 & 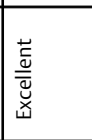 & \begin{tabular}{|l} 
\\
$\underline{\underline{\underline{\omega}}}$ \\
$\underline{\tilde{u}}$ \\
$\dot{x}$
\end{tabular} & 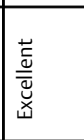 & 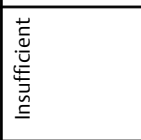 & 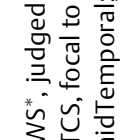 \\
\hline 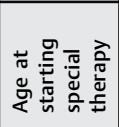 & 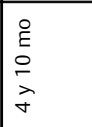 & 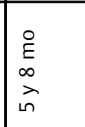 & \begin{tabular}{|l}
$\stackrel{̊}{E}$ \\
$亡$ \\
\\
n
\end{tabular} & \begin{tabular}{|l}
$\stackrel{O}{E}$ \\
$\hat{E}$ \\
\\
n
\end{tabular} & 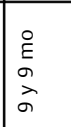 & 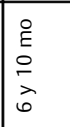 & 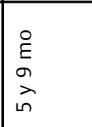 & 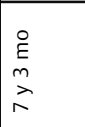 & 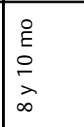 & 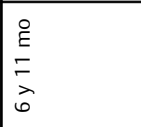 & 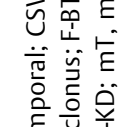 \\
\hline 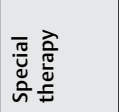 & 至 & İ & 豆 & 全 & 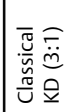 & 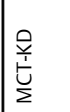 & 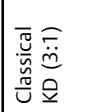 & 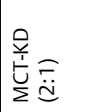 & 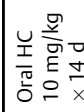 & 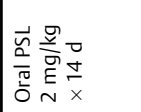 & 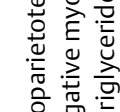 \\
\hline 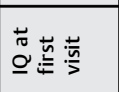 & $\begin{array}{l}0 \\
0 \\
\mathscr{a}^{\prime}\end{array}$ & $\bar{\varrho}$ & $\infty$ & $\infty$ & & $\stackrel{\stackrel{n}{=}}{=}$ & 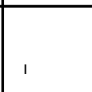 & 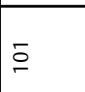 & & $\begin{array}{l}\infty \\
0 \\
0 \\
0\end{array}$ & $=\bar{a}$ \\
\hline 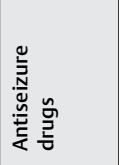 & 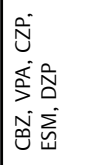 & 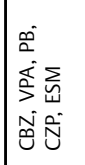 & 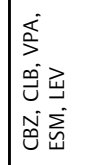 & 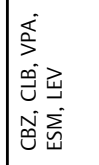 & 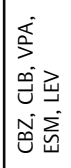 & 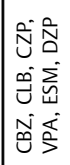 & 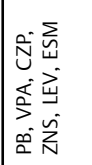 & 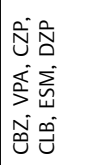 & 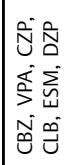 & 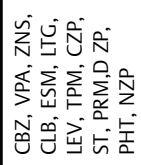 & 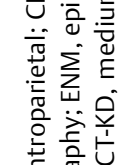 \\
\hline 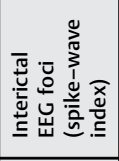 & 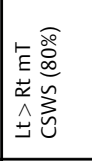 & 离 & 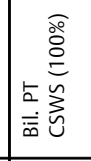 & 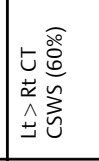 & $\begin{array}{l}0 \\
0 \\
\pm \\
\pm\end{array}$ & 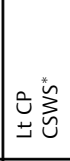 & 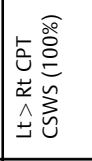 & 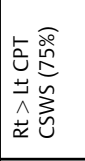 & 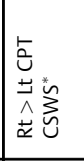 & 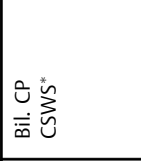 & 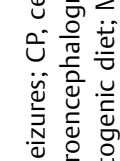 \\
\hline 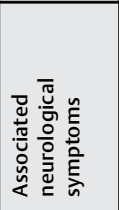 & 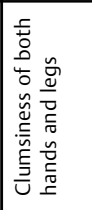 & 1 & 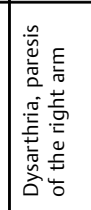 & 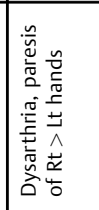 & I & 1 & 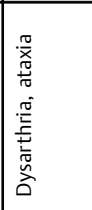 & I & 1 & 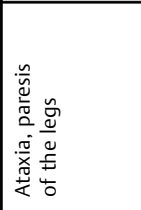 & 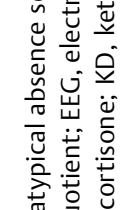 \\
\hline 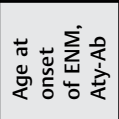 & 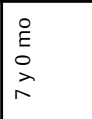 & 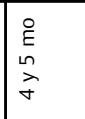 & 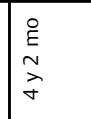 & 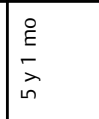 & & \begin{tabular}{|l|} 
O \\
E \\
in \\
in
\end{tabular} & 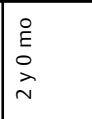 & 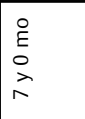 & & 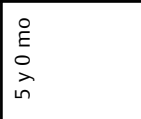 & 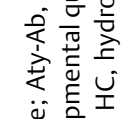 \\
\hline 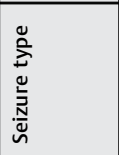 & 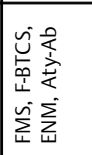 & 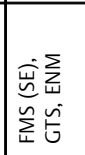 & 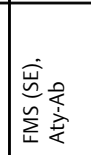 & 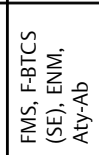 & 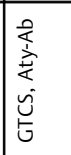 & 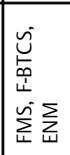 & 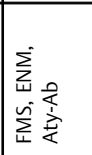 & 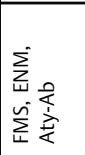 & 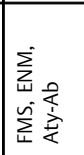 & 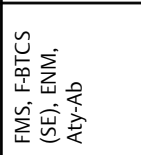 & 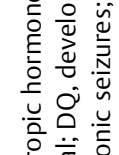 \\
\hline ڤ్ & $\frac{\frac{0}{\pi}}{2}$ & \begin{tabular}{|l|}
$\frac{0}{\pi}$ \\
$\frac{\tilde{E}}{\tilde{E}}$ \\
\end{tabular} & $\frac{0}{\frac{\pi}{\pi}}$ & 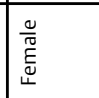 & & 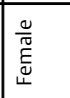 & $\frac{\frac{0}{\tilde{m}}}{\stackrel{2}{\Sigma}}$ & 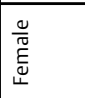 & & 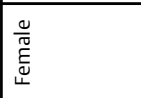 & 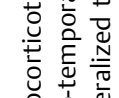 \\
\hline 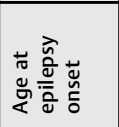 & \begin{tabular}{|l}
0 \\
$E$ \\
0 \\
$\grave{m}$ \\
$m$
\end{tabular} & 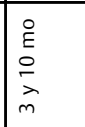 & 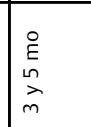 & 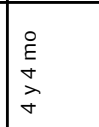 & & 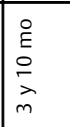 & \begin{tabular}{|l}
0 \\
E \\
0 \\
ते \\
N
\end{tabular} & 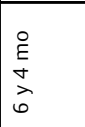 & & 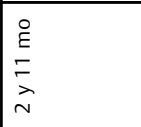 & $\overline{\bar{\tau}} \overline{\bar{U}}$ \\
\hline 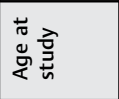 & $\underset{\text { dे }}{\text { a }}$ & $\vec{J}$ & $\begin{array}{l}\vec{\lambda} \\
=\stackrel{0}{E}\end{array}$ & 음 & & $\stackrel{2}{\circ}$ & aे & 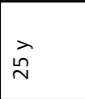 & & $\vec{E}$ & \\
\hline $\begin{array}{l}\dot{0} \\
\dot{c} \\
\dot{n}\end{array}$ & - & N & $\mathrm{m}$ & + & & in & 0 & $\wedge$ & & $\infty$ & 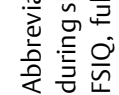 \\
\hline
\end{tabular}


function was suggested by IQ at the last follow-up period in six out of patients. The patients underwent the specific therapies at ages ranging from 58 to 117 months, with a median of 76 months. Regarding treatment, ACTH therapy $(n=4)$, KD therapy $(n=4)$, and oral steroid therapy $(n=2)$ were tried for the eight patients. Two patients (cases 4 and 7 ) tried ACTH or oral steroid and KD therapies because of an unsatisfactory response to the first therapy. In summary, excellent short-term and long-term responses were achieved by four of four patients undergoing ACTH therapy. In the KD therapy group, excellent short-term and long-term effects were observed in three out of four and two out of three patients, respectively. In the oral steroid therapy group, one patient had excellent shortterm and long-term responses, whereas another exhibited inadequate short-term and long-term effects. There have been no significant side effects of the specific therapies during the clinical course.

\section{Discussion}

In patients with ABPE, ESM in combination with VPA has been demonstrated to be effective for both ENM and CSWS. ${ }^{7}$ However, appropriate ASDs are not always introduced because focal seizures and F-BTCS always precede or develop at the same time as ENM and atypical absence seizures. The patients may develop a peculiar form of nonconvulsive status epilepticus associated with ENM. ${ }^{8}$

ACTH and oral steroid therapies have been used not only for infantile spasms but also Lennox-Gastaut's syndrome, ${ }^{9}$ epilepsy with myoclonic-atonic seizures, ${ }^{10}$ epilepsy with CSWS, ${ }^{11}$ and Landau-Kleffner's syndrome, ${ }^{12}$ although it is unclear whether ACTH is effective for patients without infantile spasms. ${ }^{13}$ In our four patients, ACTH therapy had excellent short-term and long-term effects for not only ENM and CSWS but also for focal seizures and FBTCS. As demonstrated in case 4, ACTH may be effective for a few years, but the seizures and CSWS then relapse to the previous state, requiring another specific therapy. This is reasonable considering the self-limiting natural course of ABPE in which the ABPE epileptogenesis itself gradually slows and remits around adolescence. ${ }^{5}$ Thus, patients with resistant disease such as those in cases 4 and 6 were expected to have seizure and CSWS recurrence because they were young, whereas patients with milder cases entered remission following one short course of ACTH therapy or KD therapy.

High-dose oral steroid therapy as a substitute for ACTH has been frequently used outside Japan, although it is less effective. ${ }^{14}$ of our patients, one exhibited an excellent response, whereas another exhibited an unsatisfactory response. In Japan, it is difficult to use high-dose ( $>2 \mathrm{mg} / \mathrm{kg}$ ) oral prednisolone for a long period ( $>2$ weeks) due to the lack of an official indication and the risk of side effects on an outpatient basis. Thus, initial ACTH therapy may be beneficial for patients with ABPE whose condition is refractory to ASDs if the socioeconomic situation allows.

Two of the four patients received successful KD therapy, although the seizures and CSWS transiently relapsed 2 years after KD therapy was stopped in one patient. There have been conflicting results regarding the effectiveness of KD therapy for epileptic encephalopathy with CSWS. ABPE itself may be a less refractory type of epilepsy with CSWS because the seizures and CSWS in most patients have been reported to respond favorably to ESM. In addition, in our experience, the spike-wave index of CSWS was between 50 and $85 \%{ }^{5}$ Thus, ABPE may have a better epilepsy prognosis than other CSWS syndromes. ${ }^{11,15}$ of note, one previous genetic study demonstrated that GRIN2A mutations are a major risk factor causing RE, in which ABPE was found to have an intermediate GRIN2A mutation rate $(10.0 \%)$ between that of typical RE (5.6\%) and CSWS/Landau-Kleffner's syndrome $(17.6 \% / 25.0 \%){ }^{16}$

Although the number of pharmacoresistant ABPE patients was insufficient to make a definitive conclusion, they often had frequent and repeated prolonged focal seizures or F-BTCS, in addition to ENM, atypical absence seizures and a spike-wave index of CSWS exceeding 85\%.

One limitation was the small number of patients undergoing the specific therapies, but they may be useful for patients with pharmacoresistant ABPE to prevent cognitive decline due to the persistence of epileptic encephalopathy. Further studies are warranted to confirm their efficacy and safety.

Note

The authors confirm that this report complies with the journal's guidelines regarding issues pertaining to ethical publication.

\section{Conflict of Interest}

None declared.

\section{References}

1 Aicardi J, Chevrie JJ. Atypical benign partial epilepsy of childhood. Dev Med Child Neurol 1982;24(03):281-292

2 Oguni H, Sato F, Hayashi K, Wang PJ, Fukuyama Y. A study of unilateral brief focal atonia in childhood partial epilepsy. Epilepsia 1992;33(01):75-83

3 Oguni H, Uehara T, Tanaka T, Sunahara M, Hara M, Osawa M. Dramatic effect of ethosuximide on epileptic negative myoclonus: implications for the neurophysiological mechanism. Neuropediatrics 1998;29(01):29-34

4 Hirano Y, Oguni H, Osawa M. Epileptic negative drop attacks in atypical benign partial epilepsy: a neurophysiological study. Epileptic Disord 2009;11(01):37-41

5 Fujii A, Oguni H, Hirano Y, Osawa M. Atypical benign partial epilepsy: recognition can prevent pseudocatastrophe. Pediatr Neurol 2010;43(06):411-419

6 Capovilla G, Beccaria F, Veggiotti P, Rubboli G, Meletti S, Tassinari CA. Ethosuximide is effective in the treatment of epileptic negative myoclonus in childhood partial epilepsy. JChild Neurol 1999; 14(06):395-400

7 Inutsuka M, Kobayashi K, Oka M, Hattori J, Ohtsuka Y. Treatment of epilepsy with electrical status epilepticus during slow sleep and its related disorders. Brain Dev 2006;28(05): 281-286

8 Ohtsuka Y, Sato M, Oka E. Nonconvulsive status epilepticus in childhood localization-related epilepsy. Epilepsia 1999;40(07): 1003-1010 
9 Arzimanoglou A, French J, Blume WT, et al. Lennox-Gastaut syndrome: a consensus approach on diagnosis, assessment, management, and trial methodology. Lancet Neurol 2009;8(01): 82-93

10 Oguni H, Tanaka T, Hayashi K, et al. Treatment and long-term prognosis of myoclonic-astatic epilepsy of early childhood. Neuropediatrics 2002;33(03):122-132

11 Veggiotti P, Pera MC, Teutonico F, Brazzo D, Balottin U, Tassinari CA. Therapy of encephalopathy with status epilepticus during sleep (ESES/CSWS syndrome): an update. Epileptic Disord 2012;14(01): $1-11$

12 Sinclair DB, Snyder TJ. Corticosteroids for the treatment of Landau-kleffner syndrome and continuous spike-wave discharge during sleep. Pediatr Neurol 2005;32(05):300-306
13 Mehta V, Ferrie CD, Cross JH, Vadlamani G. Corticosteroids including ACTH for childhood epilepsy other than epileptic spasms. Cochrane Database Syst Rev 2015;(06):CD005222

14 Knupp KG, Coryell J, Nickels KC, et al; Pediatric Epilepsy Research Consortium. Response to treatment in a prospective national infantile spasms cohort. Ann Neurol 2016;79(03):475-484

15 Tassinari CA, Cantalupo G, Dalla Bernardina B, et al. Encephalopathy related to status epilepticus during slow sleep (ESES) including Landau-Kleffner syndrome. In: Epileptic Syndromes in Infancy, Childhood and Adolescence. Montrouge: John Libbey Eurotext; 2012:255-275

16 Lemke JR, Lal D, Reinthaler EM, et al. Mutations in GRIN2A cause idiopathic focal epilepsy with rolandic spikes. Nat Genet 2013;45 (09):1067-1072 